\title{
Analoge und digitale Ausprägungen von >Fake- News in Wort und Bild
}

\section{Identifikationsmöglichkeiten und Vorschläge für einen konstruktiven Umgang mit falschen Fakten}

\section{Christian Schicha}

Friedrich-Alexander Universität Erlangen-Nürnberg, Institut für Theater- und Medienwissenschaft, Medienethik, Bismarckstraße 1, D-91054 Erlangen christian.schicha@fau.de

\begin{abstract}
The article deals with different forms and types of fake news in media and shows different ways to identify and classify such messages. The author presents initiatives that detect and correct false reports.
\end{abstract}

1

Die Lüge

»Wer einmal lügt, dem glaubt, man nicht.« Dieser Spruch zeigt die Dimension von Äußerungen, die aufgrund eines Täuschungsverdachtes mit negativen Konnotationen belegt sind. Die Lüge gilt als Regelverstoß und wird mit Niedertracht oder Verrat assoziiert. Es handelt sich um ein Fehlverhalten, das sanktioniert werden sollte. Ehrlichkeit hingegen gilt als Tugend, die angestrebt werden sollte.

Dabei kann es gute Gründe geben, zu lügen. Wer einen zu Unrecht Verfolgten in seinem Haus versteckt, und einer illegitimen Staatsmacht gegenüber negiert, dass dieser dort Schutz erhalten hat, lügt zwar. Dennoch kann diese Falschaussage moralisch geboten sein, sofern etwa die Rettung eines unschuldig verfolgten Juden vor der Gestapo erfolgt - hier wird auch von einer Notlüge gesprochen (vgl. Zehnpfennig 2017).

Es gibt weitere Grenzfälle, die eine Lüge rechtfertigen können: Wenn eine Frau bei einem Vorstellungsgespräch gefragt wird, ob sie schwanger ist oder einen Kinderwunsch hat, darf sie dies verneinen, selbst wenn dies nicht stimmt. 
Es geht hierbei um die Privatsphäre der Person, die aufgrund ihres Geschlechtes nicht diskriminiert werden darf: »Die Lüge zum Schutz der Privatsphäre setzt voraus, dass andere kein Recht haben, den fraglichen Sachverhalt zu erfahren. Das ist immer dann der Fall, wenn die anderen von diesem Sachverhalt nicht betroffen sind « (Dietz 2003, S. 143). In derartigen Fällen ist die Lüge sogar aus einer juristischen Perspektive erlaubt.

Wer seinen Partner anlügt, indem er ihm falsche Komplimente macht, erzählt zwar die Unwahrheit. Gleichwohl kann dieses Verhalten dazu beitragen, dass die Beziehung stabil bleibt. Wenn es sich ausschließlich um Höflichkeit und Rücksichtnahme handelt und niemand zu Schaden kommt, kann eine wohlwollende Lüge ggf. unproblematisch sein.

Insgesamt gilt aber, dass im sozialen Umgang Ehrlichkeit erwartet wird, um die Erwartung nicht zu enttäuschen, dass man sich auf andere Menschen verlassen und ihnen vertrauen kann. Dies gilt insbesondere für Berufsgruppen, die wie Ärtze_Ärtzinnen, Richter_innen und Polizisten_Polizistinnen eine große Verantwortung tragen. Von ihnen wird erwartet, dass sie die Wahrheit sagen, um Vertrauen in den Rechtsstaat zu erhalten (vgl. ebd.).

\section{$2 \quad$ Normative Ansprüche an die Medienberichterstattung}

Gleiches gilt auch für die Medienberichterstatter_innen. Von ihnen wird erwartet, dass sie wahrheitsgemäß und unabhängig berichten, gründlich recherchieren, ihre Quellen sorgfältig prüfen sowie gesellschaftlich relevante Sachverhalte aufzeigen, einordnen und angemessen bewerten (vgl. Kuhla 2017). Sie sollen ihrer Kontroll- und Kritikfunktion angemessen nachkommen und Distanz gegenüber der Politik und Wirtschaft wahren. Sie sollen der Wahrheit verpflichtet sein und die möglichen Folgen ihrer Berichte im Vorfeld selbstkritisch reflektieren.

Diese Leitlinien gelten primär für den investigativen Qualitätsjournalismus. Der Sport- und Boulevardjournalismus hingegen will primär unterhalten und folgt weniger hohen Ansprüchen. Aber auch hier gilt, dass mit lauteren Methoden gearbeitet werden sollte.

Insgesamt folgt der Journalismus in Deutschland, abgesehen von den öffentlich-rechtlichen Rundfunkanbietern, dem Markt- und nicht dem Integrationsmodell. Medienanbieter müssen sich in der Regel, je nach Medium, durch Klicks, Einschaltquoten oder Verkaufszahlen finanzieren. Die öffentliche Resonanz auf die Medieninhalte bestimmt die Werbepreise und den daraus resultierenden Erfolg oder Misserfolg der Anbieter. Insofern besteht ihr 
Interesse daran, die Inhalte so zu gestalten, dass ein möglichst breites Publikum erreicht wird, um die entsprechenden Medienprodukte zu verkaufen. Diese strukturellen Bedingungen sorgen jedoch nicht dafür, dass die Verbreitung von >Fake News< automatisch zunimmt oder gar legitimiert werden kann. Vielmehr sollten Medienbetriebe ein Interesse daran haben, dass ihnen geglaubt wird. Der Warencharakter der Nachricht sollte nicht dazu führen, dass die Maxime der Gewinnorientierung der Glaubwürdigkeit schadet. Die wichtigste Ressource von Medien liegt im öffentlichen Vertrauen. Medienskandale und Lügengeschichten führen zu einem Reputationsverlust des betroffenen Mediums und langfristig zu einem negativen Image (vgl. Schicha 2015).

»Was wir von der Gesellschaft und ihrer Welt wissen, wissen wir fast ausschließlich durch die Massenmedien. Gleichzeitig haben wir jedoch den Verdacht, dass dieses Wissen manipuliert wird. Zumindest kommt es extrem selektiv zustande, gesteuert zum Beispiel durch wenige Faktoren, die den Nachrichtenwert von Informationen bestimmen oder Unterhaltungssendungen attraktiv erscheinen lassen. Aber dies Gegenwissen wirkt sich nicht aus. Die Realität ist so hinzunehmen, wie sie von den Massenmedien präsentiert und rekursiv, auf sich selbst aufbauend, reproduziert wird.« (Luhmann 1996, S. 2) Dieses mehr als 20 Jahre alte Zitat des Soziologen Niklas Luhmann drückt bereits ein grundlegendes Misstrauen gegenüber Medienmeldungen aus. Das Statement skizziert grundlegende Selektionskriterien, die bei der Auswahl von Nachrichten relevant sind.

Präsident Trump beschimpft die amerikanischen Medien seit Beginn seiner Amtszeit verbal oder per Twitter regelmäßig als >Fake News< (vgl. Krienke 2017). »Trumps Twitter-Meldungen stellen Zusammenhänge derart verkürzt, verzerrt und um entscheidende Fakten beraubt dar, dass der Mediennutzer bei der Überprüfung des Wahrheitsgehaltes auf wenig Nennenswertes stößt. Hier wird die politische Lüge nicht um eines höheren Gutes willen und mit größerem Verantwortungsbewusstsein eingesetzt, sondern aus purem Eigeninteresse, ja aus Eigenliebe. Sie dient nicht dem Wohl des Gemeinwesens, sondern beschädigt die auf Vertrauen gegründeten Demokratie.« (Zehnpfennig 2017, S. 19).

Auch journalistische Berichterstatter_innen werden aktuell in Deutschland mit Begriffen wie >Lücken- oder Systempresseく assoziiert. Ihre Arbeit wird als >Pinocciopresse $<$ verunglimpft. Sie gelten als $>$ Staats-, System- und Mainstreammedien< (vgl. Lilienthal/Neverla 2017). Es wird weiterhin der Vorwurf 
artikuliert, dass primär über globale Katastrophen und Kriegen berichtet wird, während die Alltagsprobleme der Bürger_innen in der Berichterstattung häufig nicht berücksichtigt werden. Zahlreiche weitere Institutionen neben den Medien haben in der Bundesrepublik in den letzten Jahren ebenfalls an Glaubwürdigkeit eingebüßt. Führungspersönlichkeiten aus der Politik, den Banken, dem Sport und den Kirchen haben durch Skandale zu einem quasi kollektiven Reputationsverlust der entsprechenden Institutionen beigetragen (vgl. Kepplinger 2012; Kuhla 2017). Medien geraten ebenfalls immer wieder in die öffentliche Kritik. Der Boulevardpresse wird vorgeworfen, die Persönlichkeitsrechte von Prominenten nicht zu achten. Der Reise- und Motorjournalismus muss sich gegen Vorwürfe wehren, unkritisch und zu wohlwollend über Urlaubsorte und Fahrzeuge zu berichten. Vorwürfe der PR und Schleichwerbung werden in diesem Zusammenhang ebenfalls geäußert. Gegenüber der Kriegs- und Krisenberichterstattung wird der Vorwurf artikuliert, dass die eingebetteten Reporter_innen ausschließlich positiv über die Truppen berichten, die sie begleiten und von denen sie beschützt werden. >Das erste Opfer des Krieges ist die Wahrheit<, heißt es dann. Dieser Satz stellt demzufolge eine häufig geäußerte Kritik dar, die an die Kriegs- und Krisenberichterstatter innen gerichtet wird. Konkrete Fehler im Rahmen der Ukraineberichterstattung mussten unlängst auch öffentlich-rechtliche TV-Anbieter einräumen (vgl. Krüger 2016). Insofern gibt es immer wieder berechtigte Fragen hinsichtlich der Glaubwürdigkeit der Medien gegenüber bestimmten journalistischen Branchen und aufgrund von konkreten Einzelfällen, in denen die Berichterstattung nicht korrekt war. Eine Studie der Universität Leipzig kam zu folgendem Befund: Es »wurde den Probanden der Mitte-Studie 2016 die Frage vorgelegt, ob sie von der Lügenpresse sprechen würden, wenn sie an die Medienlandschaft in Deutschland denken. $14 \%$ bejahten das. $41,2 \%$ positionieren sich gegen diesen Begriff: In Westdeutschland liegt die Ablehnung dieser Aussage bei $43,2 \%$ und damit um $10 \%$ höher als in Ostdeutschland (33,6\%). In ganz Deutschland distanzierten sich also weniger als die Hälfte der Befragten von dem Begriff, beinahe die Hälfte signalisierte mit der Antwortkategorie >teils/ teils $<$ ihre Unentschiedenheit« (Decker et al. 2016, S. 62). In weiteren Befragungen vertreten rund ein Fünftel der Deutschen die Auffassung, dass der Begriff der >Lügenpresse< gerechtfertigt ist. $72 \%$ der Befragten halten den Begriff für nicht zutreffend. Knapp 30\% geben an, dass ihr Vertrauen in die Medien in den letzten Jahren gesunken ist (vgl. Eilenberger 2017).

Seit Oktober 2014 ist der Ruf `Lügenpresse $<$ in Deutschland auf den Pegidademonstrationen, unter anderem in Dresden zu hören. Anfang 2015 wurde der Begriff zum Unwort des Jahres erklärt, da er bereits von den Nationssozialisten_Nationssozialistinnen und den RAF-Terroristen_Terroristinnen verwendet wurde (vgl. Probst 2017). 


\subsection{Spektrum, Reichweite und Typen von >Fake-News}

Beim Blick auf das Phänomen von >Fake-News` fällt auf, dass dieser Begriff in ganz unterschiedlichen Kontexten und Zusammenhängen verwendet wird. Das Spektrum reicht von Spaß- und Satiremeldungen, über frei erfundene Inhalte, falsche Tatsachenbehauptungen, Übertreibungen, Teilwahrheiten, übler Nachrede, Gerüchten bis hin zum Mobbing (vgl. Martens 2017; Selg 2017). Es werden dann falsche Zahlen, Daten und Fakten präsentiert, um Menschen $\mathrm{zu}$ amüsieren, zu verängstigen, zu diskreditieren, um radikale Ansichten zu vertreten und damit auch die öffentliche Ordnung zu stören. Als >Fake News werden zudem Meldungen bezeichnet, die bewusst aus dem Zusammenhang gerissen werden. Sie stellen eine gezielte Täuschung dar und verfolgen das Ziel der Desinformation (vgl. Wolf/Dönch 2017).

Als typische >Fake News $<-G e n r e s$ werden die politische Propaganda, der Boulevard- sowie Reise- und Technikjournalismus klassifiziert.

Sängerlaub (2017) zufolge sind >Fake-News« die Verbreitung von falschen und irreführenden Desinformationen, um Personen, Organisationen oder Institutionen zu schaden. Für ihn gehören Desinformationen dazu, die eine gezielte Verbreitung von falschen oder irreführenden Informationen enthalten, um einer Person oder Instititution zu schaden. Die Dekontextualisierung oder bewusst falsche Interpretation wahrer Informationen wird hierbei ebenso eingeschlossen, wie die Manipulation eigentlich wahrer Informationen in Form von Bildern oder völlig frei erfundenen Inhalten. Die Überspitzung von politischen Inhalten etwa im Rahmen der Satire, um Gesellschaftskritik zu üben, gehört, Sängerlaub zufolge, nicht zu den >Fake-News «. Auch nicht intendierte Falschinformationen durch journalistische Fehlleistungen, die bewusste Überspitzung von Headlines und Teasern zur Gewinnung von Leser_innen und die versehentlich falsch publizierte Meldung, die dann korrigiert wird, fallen nicht in dieses Spektrum.

Der falsch kontextualisierte Inhalt zu eigentlich korrekten Informationen die bewusst falsch interpretiert werden, fällt hingegen in den Bereich der $>$ Fake-News $<$. Hierunter fällt zum Beispiel das Selfie des syrischen Geflüchteten Anis Modamani zusammen mit Angela Merkel, der später zu Unrecht beschuldigt wurde, einer der belgischen Attentäter der Anschläge auf dem Brüsseler Flughafen zu sein (vgl. Reinsch 2017). Zu den frei erfundenen Inhalten gehört z.B. die Falschmeldung, dass im Keller einer Pizzeria in Washington ein Kinderpornoring betrieben wird, in dem auch Hillary Clinton verwickelt sei (vgl. Kuhla 2017). Beim manipulierten Inhalt werden wahre Informationen absichtlich durch Bildbearbeitung oder die Verwendung falscher Quellen verändert (vgl. Becker von Sothen 2013). 
Eine besonders große öffentliche Resonanz erhalten sogenannte Verschwörungstheorien. Dabei handelt es sich unter anderem um Beobachtungen, die nicht erklärbar sind sowie unbegründete Vermutungen, Gerüchte und Mutmaßungen. So wird z.B. immer wieder behauptet, dass die Mondlandung gar nicht stattgefunden habe, der amerikanische Präsident Kennedy von der CIA oder der Mafia umgebracht worden sei oder die Bush-Regierung für die Terroranschläge am 11.9.2001 verantwortlich gewesen sei (vgl. Harder 2010).

\subsection{Fälschungen und frei erfundene Berichte}

Die solide Recherche unter Berücksichtigung von mindestens zwei unabhängigen Quellen gilt als Basis jeder seriösen Berichterstattung. Dennoch werden frei erfundene und schlecht recherchierte Berichte immer wieder verbreitet. Diese Entwicklung zeigt sich in den folgenden Fallbeispielen als eklatantes Versagen der journalistischen Sorgfaltspflicht:

»Jimmy ist acht Jahre alt und ein Heroinabhängiger der dritten Generation, ein frühreifer kleiner Junge mit rotblondem Haar, samtig braunen Augen und Nadeleinstichen, die wie Sommersprossen die babyzarte Haut seiner dünnen braunen Arme überziehen.« So beginnt eine Reportage von Janet Cooke, die am 28. September 1980 in der >Washington Post< erschien und die Zeitung damit in eine schwere Glaubwürdigkeitskrise stürzte. Denn das drogensüchtige Kind, dessen vermeintliches Schicksal eine Welle des Mitleids auslöste, gab es in Wirklichkeit gar nicht. Erst als Cooke für ihre Story den renommierten Pulitzerpreis erhalten sollte, brach ihr Lügengebilde zusammen. Die Selbstkontrolle der >Washington Post $<$, eines der Bollwerke des investigativen Journalismus in den USA, hatte in spektakulärer Weise versagt. Die Story war diesmal frei erfunden (vgl. Kolbe 2017).

Am 28. April 1983 erschütterte die Veröffentlichung der gefälschten Hitlertagebücher die Glaubwürdigkeit des `Stern<. Es stellte sich heraus, dass die Kontrollmechanismen bei der Wochenzeitung massiv versagt hatten. Die Tagebücher waren von Konrad Kujau gefälscht worden. Als das Bundeskriminalamt den Schwindel aufdeckte, hatte der `Stern < bereits 62 Bände für 9,3 Millionen DM gekauft. Auch Medien, wie das Us-Magazin >Newsweek<, berichteten 1983 in großer Aufmachung über die Hitler-Tagebücher. Es wurde nachgewiesen, dass das Papier, auf dem Hitler seine Aufzeichnungen aufgeschrieben haben soll, mit Weißmachern versetzt wurden, die erst nach $195^{\circ}$ in der Papierherstellung zum Einsatz gekommen sind. Teile der Bücher wurden von Kujau aus historischen Dokumenten abgeschrieben (vgl. Seufert 2008; Heidemann 2009). 
Der TV-Produzent Michael Born fälschte zwischen 1990 und 1995 insgesamt 32 Fernsehbeiträge und verkaufte sie u.a. an `Stern-TV $<$ und `Spiegel-TV $<(R T L)$, $>$ Frontak (ZDF) und >Zak< (ARD). Er verdiente mit absurden Berichten über Katzenjäger, Krötendrogen und den Ku-Klux-Klan in Deutschland 350.00o DM. Born wurde zu vier Jahren Haft ohne Bewährung verurteilt. Dieses Ereignis dokumentiert zum einen die kriminelle Energie von Born und zum anderen die fehlende Gegenrecherche der beteiligten Redaktionen (vgl. Born 1997; Bickenbach 2005; Thomsen 2005; Pritzl 2006).

Der Schweizer Journalist Tom Kummer arbeitete ab 1993 als HollywoodKorrespondent u.a. für die sSüddeutsche Zeitungく (sz). Zahlreiche seiner erfundenen Interviews mit prominenten Filmstars, wie Brad Pitt, Pamela Anderson und Sharon Stone, wurden bis 1999 im Magazin der SZ als authentische Gespräche abgedruckt (vgl. Bickenbach 2005; Thomsen 2005; Kummer 2009).

Ein dramatischer Todesfall führte zu einer falschen Berichterstattung, die sowohl von Boulevard-als auch von Qualitätsmedien geführt wurde. Ein sechsjähriger Junge, dessen Vater Iraker war, kam 1997 in einem Schwimmbad in Sebnitz ums Leben. Die Mutter ging von einem rechtsradikal motivierten Verbrechen aus. Zahlreiche Medien berichteten über diese vermeintliche Straftat. Die Schlagzeilen lauteten »Neo-Nazis ertränkten Kind« in der Bild-Zeitung, »Badeunfall erweist sich als rassistischer Mord « (taz) und »Ein Kind ertränkt wie eine Katze« (sz). Es stellte sich bei näherer Prüfung jedoch heraus, dass es sich um einen Badeunfall ohne Fremdeinwirkung handelte. Offensichtlich haben hier mehrere Medien voneinander abgeschrieben, ohne den Sachverhalt selbst weiter zu recherchieren (vgl. Jogschies 2001).

Dass die Technik oft Tücken hat, erlebten Redakteure_Redakteurinnen der Stuttgarter Zeitung im Januar 2012. Auf der Homepage der deutschen Zeitung erschien eine Meldung mit der Überschrift »Merkel tritt zurück«. Eine dreiviertel Stunde lang war die Falschmeldung auf der Website zu lesen. Später entschuldigte sich die Redaktion bei ihren Leser_innen: »Dieser Text entbehrt jeder sachlichen Grundlage«, hieß es in einer Stellungnahme auf stuttgarterzeitung.de (vgl. o.V. 2012).

Medien können auch psychische Gewalt auf Opfer der Berichterstattung ausüben. Dies dokumentieren zahlreiche Beispiele, bei denen die Bild-Zeitung eine negative Rolle gespielt hat. Dieses Blatt steht nach wie vor für einen gezielten Kampagnenjournalismus und sorgt dafür, dass Personen systematisch persönlich diffamiert und diskreditiert werden (vgl. Boenisch 2007; Kepplinger 2012). Provokation und Tabuverletzungen stellen dabei zentrale Strategien dar, um die Aufmerksamkeit der Leser_innen zu erreichen. Dabei wird auch die Grenze zur Lüge, Fälschung und Manipulation überschritten. Es erfolgt eine klare, aber nicht zwingend berechtigte Zuordnung von Gut und Böse. Inhalte 
werdenübertriebenundaufgebauscht.WennetwaprominentePersönlichkeiten beschuldigt werden, andere Personen sexuell missbraucht zu haben, dominiert dieser Verdacht große Teile der Medienberichterstattung. Die öffentliche Empörung ist groß und sogenannte Experten_Expertinnen äußern medienwirksam ihre Beurteilung, noch bevor der juristische Prozess überhaupt begonnen hat oder ein Urteil gesprochen worden ist:

Der Moderator Andreas Türck wurde 2004 verdächtigt, eine Vergewaltigung verübt zu haben. >Bild< startete eine Kampagne gegen ihn. Überschriften wie »Die Sex-Akte Türck« und »Hier steht Andreas Türck ein letztes Mal im Licht« trugen dazu bei, dass der zu Unrecht erhobene Vergewaltigungsvorwurf publizistisch ausgeschlachtet wurde. Da sich die Vorwürfe nicht erhärten konnten, wurde er freigesprochen. Dennoch war seine TV-Karriere durch den Reputationsverlust aufgrund der falschen Verdächtigung im Rahmen der Berichterstattung für Jahre zerstört (vgl. Schertz/Schuler 2007).

Im Fall des Wettermoderators Jörg Kachelmann, der zunächst einer Vergewaltigung beschuldigt und 2011 freigesprochen wurde, ging eine lange Mediendebatte voraus. Während die Wochenzeitung >Die Zeit< und >Der Spiegek den Angeklagten publizistisch unterstützten, wurden in der $>$ Bunte $<$ und in der >Bild< erhebliche Vorwürfe ihm gegenüber publiziert (vgl. Kepplinger 2012).

Nach der Berichterstattung über den ehemaligen Bundespräsidenten Christian Wulff stellte sich heraus, dass die über die Medien gegen ihn erhobenen Vorwürfe der unzulässigen Vorteilsnahme seit 2011 haltlos waren. Er wurde in allen Punkten juristisch freigesprochen (vgl. Kuhla 2017).

Neben Wortbeiträgen können auch Bilder so bearbeitet und manipuliert werden, dass sie als $>$ Fake-News $<$ klassifiziert werden können.

\subsection{Fake-Bilder}

Bilder besitzen als visuelle Informationsträger von Sachverhalten ein hohes Wirkungspotenzial. Ihre suggestive Kraft kann dazu führen, dass eine kritische Distanz gegenüber den angebotenen Motiven verloren geht. Das gilt vor allem dann, wenn den Rezipienten_Rezipientinnen die Kompetenz fehlt, die visuelle Logik von Bildbearbeitungen zu entschlüsseln.

Es liegt zunächst nahe, der Fotografie eine höhere Objektivität zuzuschreiben als der Malerei. Die Kamera leistet eine angebliche Übertragung der Realität des Objektes mit Hilfe des Objektivs, so dass schließlich ein fertiges Foto entsteht. Bilder bieten dennoch kein authentisches Abbild der Welt. Schon die Auswahl des Motivs, die Bildgestaltung und der gewählte Bildausschnitt hängen von den jeweils subjektiven Präferenzen, Interessen und Sachzwängen des Fotografen_der Fotografin ab. Ein Bildausschnitt wird aus einem breiten Zusammenhang gerissen. Die Perspektive, der Blickwinkel und der 
Zeitpunkt der Aufnahme spielen eine wichtige Rolle bei der Auswahl. Auch die Dreidimensionalität des realen Gegenstandes kann durch die Fotoaufnahme nicht abgebildet werden. Gleichwohl kann ggf. von einer Ähnlichkeit zwischen dem Bild und dem abgebildeten Objekt gesprochen werden. Die Fotoaufnahme verweist auf ein Referenzobjekt, das eine spezifische Bedeutung besitzt. Im Fall sogenannter realistischer Bilder kann es also einen unmittelbaren Wirklichkeitsbezug geben, der das Aussehen des Gegenstandes in einer ähnlichen Form einfängt (vgl. Lobinger 2012).

Das Misstrauen gegenüber dem Wahrheitsgehalt der Fotografie hat eine lange Tradition. Bilder in totalitären Regimen wurden bereits seit dem Beginn des 20. Jahrhunderts regelmäßig gefälscht. Politische Gegner_innen der amtierenden Machthaber_innen wurden dabei häufig wegretuschiert (vgl. Jaubert 1989; Miener 2004). Die Manipulation von Bildmaterial bedeutet die mit einer Täuschungsabsicht verbundende intentionale Veränderung von Informationen durch Auswahl, Zusätze oder Auslassungen.

Aus einer journalistischen Perspektive lassen sich kommerzielle Interessen, persönliche Profilierung, die Erfüllung von scheinbaren Rezeptionsbedürfnissen sowie die mangelnde oder oberflächliche Recherche aus Aktualitätsdruck als mögliche Gründe aufzeigen, um Bilder zu verändern. Insgesamt lassen sich u.a. folgende Techniken aufzeigen, die Bildmanipulationen ermöglichen:

- Löschen bzw. Einfügen von Bildelementen,

- die strategische Wahl der Perspektive des Aufnahmestandpunktes,

- Veränderung durch Helligkeit, Schärfe, Kontrast,

- Fotoverwendung aus anderen Kontexten,

- falsche Beschriftung,

- Ästhetisierung,

- Fotokombinationen,

- Fotomontage,

- gestellte Aufnahmen,

- Retusche,

- digitale Bearbeitung.

Kombinationen dieser Punkte sind natürlich möglich. Fotos werden schließlich ebenso wie Texte redigiert. Häufig gilt, dass Bildmanipulationen nur durch eine Gegenüberstellung von Original und Kopie erkennbar sind (vgl. Schreitmüller 2005; Becker von Sothen 2013). Dabei gilt: Bildmotive werden durch Informanten_Informantinnen, Rechercheure_Rechercheurinnen, Reporter_ innen, Fotografen_Fotografinnen, Journalisten_Journalistinnen, Agenturen, Cutter_Cutterinnen, Radakteure_Redakteurinnen und Verleger_innen ausgewählt und ggf. bearbeitet. Diese bringen ihre Interessen und Sichtweisen 
ein und können dadurch ein Bild sukzessiv in der Form verändern, dass der Betrachter_die Betrachterin manipuliert wird.

Bildmanipulationen sollen für den_die Leser_in erkennbar sein. Die Selbstverpflichtung zur Kennzeichnung durch das Wort >Montage< oder ein Zeichen, welches den Buchstaben $>\mathrm{M}<$ enthält, ist bei Fotoagenturen bereits gängige Praxis.

Insgesamt sind die Grenzen zwischen Retusche und unerlaubter Bearbeitung schwer zu ziehen, und es wird nicht immer benannt, welche konkreten Ergänzungen bei visuellen Motiven vorgenommen worden sind. Die Veränderung des Bildausschnittes oder die Verdunklung des Fotos gelten nicht als Montage und die Verknüpfung von unterschiedlichen Motiven zu einem Bild wird vielfach nicht kenntlich gemacht. Insofern sind eigentlich weitere Ausdifferenzierungen von Montagehinweisen erforderlich, um dieses Problem zu lösen (vgl. Schicha 2007).

\subsection{Zur Verbreitung von $>$ Fake-News< über das Internet}

»Fake News können in allen Medienangeboten als Text, Ton, Bild bzw. Video auftreten. Besonders prädestiniert für ihre Verbreitung ist das Internet mit seinen vielfältigen Informations- und Kommunikationskanälen.« (Selg 2017)

Die Gründe hierfür sind vielfältig. Zum einen ist der Informationsaustausch im Netz quantitativ deutlich höher als über die konventionellen Rundfunkund Printkanäle. Darüber hinaus sind im Internet neben den klassischen journalistischen Gatekeepern auch zahlreiche weitere Akteure_Akteurinnen in Blogs oder sozialen Netzwerken aktiv, die unter falschen Identitäten publizistisch tätig sind. In vielen Fällen kann nicht überprüft werden, von wem welche Inhalte stammen und welche Absichten damit verfolgt werden. Ein klassisches Impressum, das die Verantwortlichkeit für Veröffentlichungen transparent macht, ist vielfach nicht mehr vorhanden. Darüber hinaus ist es sehr einfach und kostengünstig, Inhalte aller Art über die zahlreichen Kanäle der Neuen Medien, unabhängig vom Wahrheitsgehalt, zu verbreiten. Die traditionellen Medienselbstkontrollinstanzen, wie der Deutsche Presserat (2015), sind schon aufgrund der Vielzahl der Falschmeldungen nicht in der Lage, diese Massen an Beiträgen entsprechend zu dokumentieren und zu bewerten. Hinzu kommt eine hohe Affinität der Mediennutzer_innen, gerade die Nachrichten besonders aufmerksam zu rezipieren, die überraschend spannend, skurril, unterhaltsam oder provokativ sind. Hierbei handelt es sich aber gerade um Meldungen, die z.T. frei erfunden sind. Sängerlaub (2007) zeigt eine Reihe von weiteren Gründen auf, die dazu führen, dass >Fake-News< im Netz quantitativ immer häufiger auftauchen. Schließlich ist die Zahl der Kanäle, aus denen Menschen Nachrichten beziehen können, im digitalen Zeitalter drastisch angestiegen. Social-Media-Plattformen aber auch Blogs, Foren, 
Videoportale oder Suchmaschinen, stellen zusätzliche Quellen für Meldungen dar. Während die professionellen Berichterstatter_innen lange ungefiltert über gesellschaftlich relevante Bereiche wie die Politik informiert haben, ist es heute ebenso möglich, sich via Twitter oder Facebook direkt bei den Kandidaten Kandidatinnen, Parteien, Gruppierungen oder durch anderen elektronische Organisationen zu informieren. Zudem hat die strukturelle und finanzielle Krise des Journalismus, der heute mehr Plattformen bedienen muss, aber weniger Kapazitäten und nur bedingt erfolgreiche Erlösmodelle hat, dazu geführt, dass alternative Inforationswege genutzt werden. Zudem sind die Mediennutzer_innen aktuell selbst nicht nur Konsumenten_Konsumentinnen, sondern als sogenannte >Prosumer_innen< auch Produzenten_Produzentinnen von Information. Dabei sind diese Meldungen fast ohne Kosten einfach herstellbar.

Reinbold (2017) vertritt die Auffassung, dass Falschinformationen absichtlich so produziert werden, um die Logiken der sozialen Medien auszunutzen. Das Ziel besteht in einer maximalen viralen Verbreitung, wobei sich hierfür emotionale Reflexe wie die Empörung und Reizthemen zu den Schwerpunkten Flucht, Missbrauch sowie Krieg und Frieden besonders gut eignen, um eine hohe Aufmerksamkeit zu generieren. $>$ Fake News $<$ werden vor allem aus finanziellen und aus politischen Interessen heraus verbreitet. Für die Internetplattformen und sozialen Netzwerke sind häufig abgerufene Falschnachrichten ein gutes Geschäft, weil Klicks im Netz Werbeeinnahmen bedeuten und Beiträge, die z.B. bei Facebook zahlreich geteilt werden, noch mehr Reichweite erzeugen.

Wie leicht Falschinformation zu enttarnen ist, spielt für ihre Verbreitung oft nur eine Nebenrolle. Gerade absurde Lügengeschichten können im Netz Millionen erreichen. Die klassischen Medien haben längst ihre Gate-Keeper-Funktion verlassen. Im Internet äußern sich die einstmals passiven Rezipienten_Rezipientinnen inzwischen aktiv über Blogs oder soziale Netzwerke. Dabei gilt das Motto: »Je reißerischer die Überschrift, je lauter das Foto, je spannender der Teaser, umso mehr Klicks« (Kuhla 2017, S. 41). Die Aufmerksamkeit über das Internet generiert schließlich die Werbeeinnahmen. Die Glaubwürdigkeit gerät dabei bisweilen in den Hintergrund.

Der Blogger Sascha Lobo modifiziert das bereits erwähnte Luhmann-Zitat aufgrund der sich durch das Internet ergebenen Konsequenzen wie folgt: Whas wir über die Welt wissen, wissen wir aus einem kleinen Bildschirm, der uns sozial, redaktionell und algorithmisch aufbereitete Informationen präsentiert, dabei Sensationalisiertes, Zugespitztes, Radikales tendenziell bevorzugt, was durch die Echokammern der Netzöffentlichkeit selbstverstärkend wirkt « (Lobo 2017). 
Aufgrund dieser Entwicklungen im Web 2.o hat sich also das Misstrauen speziell gegenüber der digitalen Medienberichterstattung noch erhöht.

\section{$4 \quad$ Wie lassen sich >Fake News` aufdecken und bekämpfen?}

Bereits existierende Suchmaschinen wie www.hoaxsearch.com, die sich auf Fakes im Internet spezialisiert haben, leisten wertvolle Aufklärungsarbeit. In vielen Fällen reicht aber bereits der gesunde Menschenverstand aus, um $>$ Fake News $<$ zu erkennen und zu beurteilen. Die Prüfung der Seriosität von den angegebenen Quellen und die eigene Recherche des Themas stellen hierfür einen ersten konstruktiven Ansatz dar (vgl. http://www.mimikama.at/).

Um >Fake News $<$ zu erkennen, sollte bei der Prüfung von Texten darauf geachtet werden, sich auf Details zu konzentrieren. Namen von Personen oder Organisationen sollten im Internet gesucht werden. Sofern keine oder nur lückenhafte Informationen zu finden sind, kann dies darauf hinweisen, dass sie gar nicht existieren. Um die Glaubwürdigkeit zu steigern, verweisen Falschmeldungen auch häufig auf etablierte Medien. Sofern der Text Links enthält, sollte immer die Originalquelle gelesen werden. Wenn der Verweis ins Nichts führt oder der zitierte Text eine ganz andere inhaltliche Ausrichtung hat, ist die Seriosität der verweisenden Quelle fragwürdig. Grundsätzlich gilt, dass seriöse Webseiten ihre Herkunft nicht verschleiern. Insofern sollten dort immer ein Impressum oder Kontaktmöglichkeiten vorhanden sein, die überprüfbar sind. Eine Rückwärtssuche von Bildern, z.B. über Tineye, erkennt, ob ein Foto bereits woanders benutzt wurde oder ähnliche Motive im Netz aufgetaucht sind. Dort gibt der Nutzer die Bild-urL ein oder lädt das Motiv hoch. Bei vermeintlichen Live- oder Augenzeugenvideos ist eine Rückwärtssuche ebenfalls möglich. Im Bereich der Sozialen Medien wird ein weißer Haken auf blauem Untergrund nach einer Authentizitätsprüfung eingesetzt. Sowohl Facebook als auch Twitter vergeben das Icon an verifizierte Accounts, um eine Abgrenzung von Fakeangeboten vornehmen zu können (vgl. Maciej 2016). Inzwischen sind weiteren Initiativen in ganz unterschiedlichen Kontexten entstanden, die sich professionell mit der Erkennung und Bearbeitung von >Fake News< beschäftigen. Journalisten_Journalistinnen haben selbst Projekte aufgenommen, in denen rechercheerfahrene Berichterstatter_innen Medienmeldungen auf ihren Wahrheitsgehalt prüfen. Dazu gehören u.a. die ARD-Tagesschau-Faktenfinder, die seit April 2017 tätig sind und ein Team des Bayrischen Rundfunks, das einen Monat später die Arbeit aufgenommen hat (vgl. Wolf/Dönch 2017). Fortlaufend aktualisierte Informationen zum Thema liefert auch die $>$ Bundeszentrale für politische Bildung< (vgl. bpb.de/fake-news), die auch ein 
Onlinegame (www.fakeittomakeit.de) zum Thema herausgebracht hat, sowie die Wochenzeitung >Die Zeit< (www.zeit.de/themen/fake-news), die einen $>$ Faktomat< (www.zeit.de/serie/faktomat) entwickelt hat. Unterrichtsmaterialien zu >Fake News< sind u.a.

- vom >Bayrischen Rundfunk ( www.br.de/sogehtmedien/stimmt-das/luegen -erkennen/unterrichtsmaterial-un-wahrheiten-luegen-erkennen-10o .html),

- von der >Bundeszentrale für politische Bildung « (w.bpb.de/lernen/digitale -bildung/medienpaedagogik/243064/fake-news),

- von der EU-Initiative für mehr Sicherheit im Netz >klicksafe.de< (http:// www.klicksafe.de/service/aktuelles/news/detail/stop-fake-news-neue -unterrichtsmaterialien-und-app-zum-thema/),

- vom 〉JFF-Institut für Medienpädagogik (http://www.jff.de/jff/aktivitaeten/ praxis/artikel/art/unterrichtsmaterialien-zum-thema-fake-news-und -social-bots-im-digitalen-zeitalter/),

- vom >Medienpädagogik Praxisblog $<$ (https://www.medienpaedagogik-praxis .de/2017/02/o2/fakenews-im-unterricht/),

- und von der >Landesanstalt für Medien Nordrhein Westfalen (LfM)< (http:// www.medienscouts-nrw.de/tag/fake-news/) entwickelt worden.

Des Weiteren arbeitet das interdisziplinär ausgerichtete Forschungsprojekt DORIAN (Desinformationen aufdecken und bekämpfen), das vom Bundesministerium für Bildung und Forschung seit August 2017 gefördert wird, an diesem Thema. Der Fachbereich Informatik des Frauenhofer Institutes in Darmstadt erarbeitet Verfahren der Bild- und Textforensik zur automatischen Erkennung von Falschnachrichten. Juristen_Juristinnen der Universität Kassel prüfen die rechtlichen Optionen des Vorgehens gegen $>$ Fake News Psychologen_Psychologinnen der Universität Duisburg-Essen analysieren, wie Menschen Falschinformationen wahrnehmen und darauf reagieren. Journalismus-Experten_Expertinnen der Hochschule für Medien in Stuttgart erstellen ein Konvolut von >Fake News< und ordnen diese Meldungen systematisch ein (vgl. Frauenhofer Institut 2017).

Vor allem die digitalen Medien bieten die Möglichkeit, Diskurse ohne großen technischen und finanziellen Aufwand zu bewerkstelligen. Prinzipiell kann sich jeder mit entsprechender Medienkompetenz an Debatten auf ganz unterschiedlichen Plattformen artikulieren. Dies ist im Grunde positiv zu 
bewerten. Gleichwohl ist aber auch die Fülle der destruktiven, zum Teil anonymisierten, Kommentare angestiegen. Phänomene wie >Cybermobbing<, >Shitstorm oder auch >Fake News nehmen rasant zu und richten einen erheblichen Schaden an. Populistische Narrative erzeugen eine hohe Aufmerksamkeit. Sie sorgen für hohe Klickraten, die aus einer ökonomischen Perspektive der Plattformbetreiber zunächst wünschenswert sind. Hier ist das Misstrauen in Medieninstitutionen bisweilen durchaus berechtigt. Wenn es dort Fehler oder Versäumnisse gibt, sollten diese aufgezeigt und öffentlich transparent gemacht werden. Diese Institutionen sollten auf berechtigte Kritik angemessen reagieren, Fehler zugeben und Fehlentwicklungen abstellen. Nur so kann Vertrauen wiederhergestellt werden. Insgesamt erreichen provozierende Statements und absurde Behauptungen eine hohe Medienaufmerksamkeit. Hier sind auch Medien gefordert, nach der Maxime `Gründlichkeit statt Schnelligkeit $<\mathrm{zu}$ arbeiten und eine fundierte Recherche zu leisten, bevor sie Nachrichten und Meldungen veröffentlichen, die nicht stichhaltig sind. Immer wenn Falschmeldungen publiziert werden, trägt dies zum Reputationsverlust von Medien bei. Vorurteile gegenüber der sogenannten Lügenpresse werden dadurch verstärkt und fördern auch populistische Tendenzen.

Gleichwohl ist nicht alles was falsch ist als $>$ Fake News $<$ zu klassifizieren. Es ist wichtig, zwischen einer extremen Zuspitzung, Verzerrung, Satire und tatsächlichen Falschmeldungen zu differenzieren. Medien aller Gattungen machen zudem Fehler in der Berichterstattung. Wichtig ist immer eine Analyse der Motive, die dazu führen, dass $>$ Fake News $<$ verbreitet werden. So stellt sich z.B. die Frage, ob mangelhafte Recherche dafür verantwortlich ist, dass unwahre Behauptungen publiziert werden, oder ob eine Person oder Institution gezielt und bewusst diffamiert werden soll.

Für die moralische und juristische Bewertung von $>$ Fake News zunächst die gesellschaftlichen Umstände und Rahmenbedingungen, unter denen die Medienanbieter agieren, zu berücksichtigen, um Handlungsspielräume und Rahmenbedingungen für eine Medienberichterstattung aufzuzeigen, die ethischen Leitlinien folgen. Gleichwohl sollten auch die Internetplattformen und sozialen Netzwerke wie Google oder Facebook in die Verantwortung genommen werden, schon aus Eigeninteresse wirksame Strategien gegen Falschmeldungen aller Art zu entwickeln, da auch die Reputation dieser Unternehmen leidet, wenn dort regelmäßig unwahre Meldungen verbreitet werden. Kurzfristig mag es zwar ein lukrativer Geschäftsanreiz sein, möglichst viele Klicks der Nutzer - unabhängig vom Wahrheitsgehalt - zu erreichen. Langfristig ist diese Strategie jedoch kontraproduktiv, da sich die Kunden_Kundinnen von unseriösen Anbietern ggf. abwenden werden (vgl. Schicha 2016a; 2016b; 2017). 


\section{Literatur}

Becker von Sothen, Hans (2013): Bild-Legenden. Fotos machen Politik. FälschungenFakes-Manipulationen. Graz: Ares.

Bickenbach, Matthias (2005): Der Fälscher als Poetologie und Medientheoretiker. Ein Entwurf im außermoralischen Sinn über Michael Borns und Tom Kummers Werkstattberichte der Fernsehwirklichkeit. In: Claudia Gerhards/Stephan Borg/Bettina Lambert (Hrsg.): TV-Skandale. Konstanz: UVK, S. 329-354.

Boenisch, Vasco (2007): Strategie: Stimmungsmache. Wie man Kampagnenjournalismus definiert, analysiert - und wie ihn die BILD-Zeitung betreibt. Köln: Herbert von Halem.

Born, Michael (1997): Wer einmal fälscht ... Die Geschichte eines Fernsehjournalisten. Köln: Kiepenheuer \& Witsch.

Dietz, Simone (2003): Die Kunst des Lügens. Eine sprachliche Fähigkeit und ihr moralischer Wert. Reinbek bei Hamburg: Rowohlt.

Eilenberger, Wolfram (2017): Und woran zweifelst Du? Leitfaden für das postfaktische Zeitalter. In: Philosophie Magazin 3/2017, S. 40-43.

Harder, Bernd (2010): Elvis lebt! Lexikon der unterdrückten Wahrheiten. Freiburg i. Br.: Herder.

Heidemann, Gerd (2009): Der Nazi-Tick. In: Jens Bergmann/Bernhard Pörksen (Hrsg.): Skandal! Die Macht öffentlicher Empörung. Köln: Herbert von Halem, S. $143^{-151 .}$

Jaubert, Alain (1989): Fotos, die lügen. Politik mit gefälschten Bildern. Frankfurt/Main: Athenäum.

Jogschieß, Rainer (2001): Emotainment. Journalismus am Scheideweg. Der Fall Sebnitz und die Folgen. Münster: LIT.

Kepplinger, Hans Matthias (2012): Die Mechanismen der Skandalisierung. Zu Guttenberg, Kachelmann, Sarrazin \& Co: Warum einige öffentlich untergehen und andere nicht. München: Olzog.

Krienke, Patrick von (2017): Alles \#FakeNews, oder was. In: if. Zeitschrift für Innere Führung 3/2017, S. 22-28.

Krüger, Uwe (2016): Mainstream - Warum wir den Medien nicht mehr trauen. München: C.H. Beck.

Kuhla, Karoline (2017): Fake News. Hamburg: Carlsen.

Kummer, Tom (2009): Faction. In: Jens Bergmann/Bernhard Pörksen (Hrsg.): Skandal! Die Macht öffentlicher Empörung. Köln: Herbert von Halem, S. 196-207.

Liebert, Wolf-Andreas/Metten, Thomas (Hrsg.) (2007): Mit Bildern lügen. Köln: Herbert von Halem.

Lilienthal, Volker/Neverla, Irene (Hrsg.) (2017): Lügenpresse. Anatomie eines politischen Begriffs. Köln: Kiepenheuer \& Witsch. 
Lobinger, Katharina (2012): Visuelle Kommunikationsforschung. Medienbilder als Herausforderung für die Kommunikations- und Medienwissenschaft. Wiesbaden: Springer vs.

Luhmann, Niklas (1996): Die Realität der Massenmedien (2. Aufl). Opladen: Westdeutscher Verlag.

Miener, Frank (2004): Bilder, die lügen. Norderstaedt: Book on Demand.

Pritzl, Thomas (2006): Der Fake-Faktor. Spurensuche im größten Betrugsfall des deutschen Fernsehens. München: Kopaed.

Schertz, Christian/Schuler, Thomas (2007): Rufmord und Medienopfer. Die Verletzung der persönlichen Ehre. Hamburg: Ch. Links.

Schicha, Christian (2007): Manipulierte Fotos oder ein neues Gesicht für Angela Merkel. In: Simone Dietz/Timo Skrandies (Hrsg.): Mediale Markierungen. Studien zur Anatomie medienkultureller Praktiken. Bielefeld: transcript, S. 155-182.

Schicha, Christian (2016): Die falsche Spur ist oft interessanter. Interview mit Frank Stecher. In: Nürnberger Zeitung vom 20. Dezember. [= Schicha 2016b]

Schreitmüller, Andreas (2005): Alle Bilder lügen. Foto-Film-Fernsehen-Fälschung. Konstanz: UVK.

Seuffert, Michael (2008): Der Skandal um die Hitler-Tagebücher. Frankfurt/Main: Fischer.

Thomsen, Holger (2005): Fälschung und Qualitätssicherung im Journalismus. Michael Born und Tom Kummer über die skandalöse Normalität des Fernsehens. In: Claudia Gerhards/Stephan Borg/Bettina Lambert (Hrsg.): TV-Skandale. Konstanz: UVK, S. $355^{-}-372$.

Wolf, Susanna/Dönch, Katharina (2017): Fakt oder Fake. In: www.didacta-magazin 4/2017, S. 30-33.

Zehnpfennig, Barbara (2017): Nichts als die Wahrheit. In: ZEITGeschichte 3/2017, S. 14-19.

\section{Internetquellen}

Decker, Oliver/Kiess, Johannes/Eggers, Eva/Brähler, Elmar (2016): Die »Mitte«-Studie 2016: Methode, Ergebnisse und Langzeitverlauf. In: Oliver Decker/Johannes Kiess/ Elmar Brähler (Hrsg.): Die enthemmte Mitte. Autoritäre und rechtsextreme Einstellung in Deutschland. Gießen: Psychosozial, S. 23-67. URL: https://www.boell.de/ sites/default/files/buch_mitte_studie_uni_leipzig_2016.pdf?dimension1=division _demo (Datum des letzten Abrufs: 10. Dezember 2017).

Deutscher Presserat (Hrsg.) (2015): Publizistische Grundsätze (Pressekodex). URL: http://www.presserat.de/pressekodex/pressekodex/\#panel-praeambel (Datum des letzten Abrufs: 10. Dezember 2017). 
Frauenhofer Institut (2017): Fake News finden und bekämpfen. URL: https://www .sit.fraunhofer.de/de/news/aktuelles/presse/details/news-article/fake-news-finden -und-bekaempfen/ (Datum des letzten Abrufs: 10. Dezember 2017).

Kolbe, Corina (2017): Historische Fake News. Die Reporterin und der achtjährige Junkie, den es nie gab. URL: http://www.spiegel.de/einestages/historische -fake-news-die-groessten-zeitungsenten-a-1166379.html (Datum des letzten Abrufs: 10. Dezember 2017).

Lobo, Sascha (2016): Das Ende der Gesellschaft. Digitaler Furor und das Erblühen der Verschwörungstheorien. In: Blätter für deutsche und internationale Politik. Oktober 2016. URL: https://www.blaetter.de/archiv/jahrgaenge/2016/oktober/das -ende-der-gesellschaft\%20\%20 (Datum des letzten Abrufs: 10. Dezember 2017).

Maciej, Martin (2016): Twitter: Account verifizieren lassen - so bekommt man den blauen Haken. URL: http://www.giga.de/webapps/twitter/tipps/twitter-account-ve rifizieren-lassen-so-bekommt-man-den-blauen-haken/ (Datum des letzten Abrufs: 10. Dezember 2017).

Martens, Rene (2017):Im Nebel.Falschmeldungen, Lügen, Fake News:WieFehl-und Desinformation betrieben wird. URL: http://www.medienkorrespondenz.de/leitartikel/ artikel/im-nebel.html (Datum des letzten Abrufs: 10. Dezember 2017).

o.V. (2012): »Merkel tritt zurück«. In: Hamburger Abendblatt vom 27.1.2012. URL: https://www.abendblatt.de/kultur-live/tv-und-medien/article107726038/ Merkel-tritt-zurueck-Stuttgarter-Zeitung-mit-peinlicher-Panne.html (Datum des letzten Abrufs: 10. Dezember 2017).

Probst, Maximilian (2017): Welt unter Druck. So alt wie die Presse ist der Vorwurf, sie verbreite Lügen und sei das Werkzeug von Verschwörern. In: ZEITGeschichte 3/2017, S. 98-101. URL: http://www.zeit.de/zeit-geschichte/2017/03/verschwoerungs theorien-presse-luegenpresse-medien (Datum des letzten Abrufs: 10. Dezember 2017).

Reinbold, Fabian (2017): Die Wahrheit über Fake News. URL: http://www.spiegel .de/netzwelt/web/donald-trump-die-wahrheit-ueber-fake-news-a-1129628.html (Datum des letzten Abrufs: 10. Dezember 2017).

Reinsch, Melanie (2017): Klage gegen Facebook. Wie ein Selfie mit Merkel das Leben eines Flüchtlings veränderte. In: Berliner Zeitung vom 3.2.2017. URL: https://www .berliner-zeitung.de/kultur/klage-gegen-facebook-wie-ein-selfie-mit-merkel -das-leben-eines-fluechtlings-veraenderte-25673026 (Datum des letzten Abrufs: 10. Dezember 2017).

Sängerlaub, Alexander (2017): Deutschland vor der Bundestagswahl: Überall Fake News?! URL: https://www.stiftung-nv.de/sites/default/files/fakenews.pdf (Datum des letzten Abrufs: 10. Dezember 2017). 
Schicha, Christian(2015):Skandale in den Medien. Strategische und ethische Überlegungen der öffentlichen Kommunikation für den Journalismus, die Politik und die Wirtschaft. URL: http://newsroom.mediadesign.de/imfokus/skandal-in-den-medien -strategische/ (Datum des letzten Abrufs: 10. Dezember 2017).

Schicha, Christian (2016): Der Reiz des Falschen - Politik fürchtet Fake News. Interview für detektor.fm. URL: https://detektor.fm/digital/politik-fuerchet-fake-news (Datum des letzten Abrufs: 10. Dezember 2017). [= Schicha 2016a]

Schicha, Christian (2017): Gegen Fake News und alternative Fakten: Wie »geht« politische Kommunikation im Zeitalter neuer Medien? Interview mit dem DSGVBlog der Sparkasse vom 27.9.2017. URL: http://blog.dsgv.de/christian-schicha-gegen -fake-news-und-alternative-fakten/ (Datum des letzten Abrufs: 10. Dezember 2017). Selg, Olaf (2017): Fake News. In: tv diskurs. Verantwortung in audiovisuellen Medien am 31.3.2017. URL: http://tvdiskurs.de/beitrag/fake-news/ (Datum des letzten Abrufs: 10. Dezember 2017). 